Rev. salud pública. 14 (2): 226-237, 2012

\title{
Fiabilidad y validez del instrumento "Fantástico" para medir el estilo de vida en adultos colombianos
}

\section{The Fantastic instrument's validity and reliability for measuring Colombian adults' life-style}

\author{
Robinson Ramírez-Vélez y Ricardo A. Agredo²
}

1 Programa de Fisioterapia, Universidad Manuela Beltrán. Bogotá, Colombia. robin640@hotmail.com

2 Centro para la Investigación en Salud y Rendimiento Humano ZOE. Escuela de Salud Púbñica. Universidad del Valle. Cali, Colombia. fitsaludintegral@gmail.com

Recibido 17 mayo 2011/Enviado para Modificación 3 Marzo 2012/Aceptado 12 Abril 2012

\section{RESUMEN}

Objetivo El cuestionario «Fantástico» fue creado para ayudar a los profesionales de la salud a medir los estilos de vida de sus pacientes. El objetivo fue evaluar la fiabilidad y validez del cuestionario Fantástico, traducido al español en la versión de 3 opciones de respuesta, en un grupo de adultos colombianos.

Materiales y Métodos Se aplicó por entrevista auto diligenciada en 550 personas. Se usaron medidas de tendencia central y dispersión para los dominios y grupos; cálculo de consistencia interna y correlación interescalar.

Resultados Se encontró que los 25 ítems- y los agrupados en los 10 dominios superaron el estándar propuesto de fiabilidad ( $\alpha$ de Cronbach) mayor a 0,50 y 0,73, respectivamente. Se encontró correlación interescalar como buenas y aceptables, en las diferentes categorías del cuestionario Fantástico (total vs. dominios), $(r=0,19-0,79 p<0,01)$.

Conclusiones La versión 3 del cuestionario Fantástico reúne algunos de los criterios de consistencia interna y validez de constructo. Se recomienda utilizar el cuestionario en la atención primaria en salud y en estudios epidemiológicos.

Palabras Clave: Estilo de vida, validez de las pruebas, reproducibilidad de resultados, adulto, Colombia (fuente: DeCS, BIREME).

\section{ABSTRACT}

Objective The Fantastic lifestyle assessment questionnaire was designed to help healthcare physicians measure their patients' life-styles. This work was thus aimed at assessing the reliability and validity of the Fantastic questionnaire (version 3 translated into Spanish) on a group of Colombian adults.

Materials and Methods The questionnaire was administered to 550 people 
to fill out themselves. Measures of central tendency and dispersion were used for the domains and groups; internal consistency and inter-scalar correlation were calculated.

Results It was found that the 25 items, and those grouped into 10 domains exceeded the proposed reliability standard (Cronbach $\alpha$ ), being greater than 0.50 and 0.73 , respectively. Inter-scalar correlation was good and acceptable in various categories of the Fantastic questionnaire (total cf domains), $(r=0.19$ to $0.79 ; p<0.01$ ).

Conclusions Fantastic questionnaire version 3 brings together some of the internal consistency and construct validity criteria. It is recommended that the questionnaire be used in primary healthcare and epidemiological studies.

Key Words: Lifestyle, test validity, results' reproducibility, adult, Colombia (source: $\mathrm{MeSH}, \mathrm{NLM})$.

$\mathrm{L}$

os estilos de vida se caracterizan por patrones de comportamiento identificables que pueden tener un efecto sobre la salud de un individuo y se relacionan con varios aspectos que reflejan las actitudes, valores y comportamientos en la vida de en una persona (15). Reaven (6), en la década de 1980, observó que la dislipidemia, la hipertensión arterial y la hiperglicemia, eran condiciones frecuentemente asociadas a un inadecuado estilo de vida. Otros autores han resaltado quesujetos que acusan conductas y preferencias relacionadas con un estilo de vida saludable como alimentación balanceada, práctica regular de actividad física, menor consumo de alcohol, tabaco u otras drogas, el riesgo de presentar patologías cardio-metabólicas era menor, al compararse con sus pares que mostraban un estilo de vida inadecuado $(4-7,8)$.

En este marco, cobra valor el debate de conocer los estilos de vida y su impacto sobre la salud, a pesar que, en la literatura actual se evidencie una gran laguna en la aplicación de instrumentos que estiman este componente. En este sentido, para conocer el comportamiento de una población en particular o proponer apoyo para modificar aquellos perjudiciales para la salud, se han diseñado cuestionarios, que identifican dichos patrones de conducta, muchos de ellos económicos y de fácil aplicación $(3,8,9)$.

El cuestionario «FANTÁSTICO» es un instrumento genérico diseñado en el Departamento de Medicina Familiar de la Universidad McMaster de Canadá, y permite identificar y medir el estilo de vida de una población particular $(10,11)$. Este cuestionario contiene 25 ítems cerrados que explora nueve categorías o dominios físicos, psicológicos y sociales relacionados al estilo de vida. Sus versiones cortas y extensas, han sido validadas en 
jóvenes estudiantes $(12,13)$, en trabajadores (14) y en pacientes de consulta clínica general (15). Ambas versiones, han sido adaptadas por un panel de profesionales de la salud para su mejor comprensión en sujetos mexicanos, españoles y brasileros (7-9).

Contar con instrumentos de este tipo, que tengan validez y fiabilidad, permitirá a los profesionales de la salud identificar de forma rápida y objetiva los factores de riesgo y pronóstico del estilo de vida que pueden ser mejorados con la intervención del equipo de salud y la participación comprometida del paciente $(9,10)$. Además, al aplicarse en forma seriada, permitirá realizar el seguimiento para evaluar el resultado de las acciones terapéuticas o educativas implementadas. El objetivo de este trabajo fue evaluar la validez y fiabilidad del cuestionario Fantástico, traducido al español en la versión de 3 opciones de respuesta, en un grupo de adultos colombianos.

\section{MATERIAL Y MÉTODOS}

Se realizó un estudio transversal, observacional de validación comunitaria en captación consecutiva entre julio de 2009 y julio de 2010. La población y muestra estuvo constituida por todas las personas mayores a 18 años $(\mathrm{n}=550)$, colombianos, de habla hispana, residentes del Departamento del Valle del Cauca, área metropolitana de la Ciudad de Cali, considerados como saludables (según encuesta de antecedentes médicos tomada de la historia clínica simplificada o que no hubieran consultado por un trastorno físico, ni mental en los últimos 3 meses) y asistían a control médico particular al Centro para la Investigación en Salud y Rendimiento Humano. Se excluyeron sujetos que respondieron en la encuesta de antecedentes personales o consulta médica en los últimos tres meses: cirugía o trauma mayor reciente, enfermedades endocrinas como (Diabetes mellitus, hipo/hipertiroidismo, obesidad mórbida (IMC $>35 \mathrm{~kg} / \mathrm{m} 2$ ), enfermedades autoinmunes como (lupus, psoriasis, enfermedades del colágeno), cáncer de cualquier etiología y alteración respiratoria/cardiaca significativa (como ICC, EPOC, neumonía, asma y bronquitis).

Antes de ser incluidos los participantes fueron informados acerca de la investigación, suministrando su consentimiento informado por escrito en forma voluntaria y recibiendo explicación sobre la evaluación del estado y percepción de la salud. La información se recolectó mediante 
entrevista personal e historia clínica simplificada, aplicación de encuesta sociodemográfica y auto-reporte del estilo de vida identificado mediante la aplicación de la versión española del cuestionario «Fantástico» (7-10).

Este cuestionario presenta tres opciones de respuesta con valor numérico de 0 a 2 para cada categoría, y se califican por medio de una escala tipo Likert, con una calificación de 0 a 100 puntos. Tomando como punto de corte la media de las calificaciones propuestas por los autores del instrumento $(9,10)$ cinco niveles de calificación estratifican el comportamiento: $(<39$ puntos $=$ existe peligro, 40 a 59 puntos $=$ malo, 60 a 69 puntos $=$ regular, 70 a 84 puntos $=$ bueno, 85 a 100 puntos $=$ excelente) estilo de vida. Cuanto menor sea la puntuación, mayor es la necesidad de cambio. En términos generales, los resultados pueden ser interpretados de la siguiente manera: "Excelente" indica que el estilo de vida del individuo representa una influencia óptima para la salud; "Bueno" indica que el estilo de vida representa una influencia adecuada para la salud; "Regular" indica que el estilo de vida representa un beneficio para la salud, aunque también presenta riesgos, "malo y existe peligro" indica que el estilo de vida del individuo plantea muchos factores de riesgo.

Para evaluar las propiedades psicométricas (16) de este instrumento se siguió el protocolo de adaptación y validez del cuestionario original y la validación en población mexicana y brasilera $(7-10,17,18)$, quienes encontraron que las escalas superaron el estándar propuesto de fiabilidad (alfa de Cronbach>0,6).

Adicionalmente, un estudio piloto fue conducido para estimar el tiempo promedio del auto-diligenciamiento del instrumento e identificar durante la actividad, el conocimiento y aclaración de algunos términos (ítems) utilizados en el cuestionario. Todo el estudio se enmarcó dentro de las disposiciones vigentes para la protección de los sujetos humanos que participan en investigación contenidas en la resolución 8430 de 1993 del Ministerio de Salud de Colombia y contempladas en la Declaración de Helsinki clasificando este estudio "con riesgo mínimo", según el numeral b del Artículo 11, previa aprobación del Comité de investigación de la FUMC, Cali.

Los resultados fueron analizados en el paquete estadístico Statistic Package for the Social Sciences (SPSS), versión 15, calculándose medidas 
de tendencia central, dispersión. La consistencia interna (fiabilidad) indica el grado en el que las preguntas incluidas en una sub-dimensión (o dominio) miden el mismo concepto. Ésta se analizó mediante el coeficiente $\alpha$ de Cronbach, considerándose aceptable para este estudio un coeficiente alfa de 0,6 o mayor según lo recomendado por los autores de España, México y Brasil y por la teoría psicométrica para la población, sexo y grupos de edad (7-10,16,17). Para comprobar la capacidad de discriminación del instrumento según las características de los individuos que participaron en el estudio, se calcularon las medias y desviación estándar (DE) de las puntuaciones de cada dominio e ítems del cuestionario por grupos de edad (18-34 años y 35-64 años) y sexo. La normalidad de las variables se evaluó mediante la prueba de Kolmogorov-Smirnov. La correlación interescalar se estimó a través del coeficiente de correlación de Pearson (r). Un valor $\mathrm{p}<0,05$ se consideró como significante.

\section{RESULTADOS}

Participaron voluntariamente un total de 554 personas, de los cuales 550 $(99,27 \%)$ respondieron completo el cuestionario y fueron incluidos en el análisis. El 74 \% (407 personas) eran de género masculino. La edad estaba comprendida entre 20 y 64 años, con una media $32,54 \pm 8,6$ en las mujeres y entre 19 y 62 años, con una media $31,21 \pm 7,79$ en los hombres.

La Tabla 1, resume los resultados del cuestionario Fantástico según la clasificación del estilo de vida en la población general $(\mathrm{n}=550)$. Se encontró que 4 sujetos $(0,7 \%)$ obtuvieron una calificación de malo (inferior a 60 puntos), 29 sujetos (5,3\%), regular (entre 60 y 69 puntos), 258 sujetos (47\%) bueno (entre 70 y 84 puntos) y 259 sujetos ( $47 \%$ ) excelente (entre 85 y 100 puntos). En este grupo, la confiabilidad del instrumento evaluado por el alfa de Cronbach fue 0,73. En hombres, se encontró que 3 sujetos $(0,7 \%)$ presentaban una calificación de malo (inferior a 60 puntos), 22 sujetos $(5,4 \%)$ regular (entre 60 y 69 puntos), 184 sujetos (45,2\%) bueno (entre 70 y 84 puntos) y 198 sujetos $(48,6 \%$ ) excelente (entre 85 y 100 puntos), con una confiabilidad por $\alpha$ de Cronbach de 0,73 . En mujeres 1 sujeto $(0,7 \%)$ se clasificó en estilo de vida malo (inferior a 60 puntos), 7 sujetos (4,9\%), estilo de vida regular (entre 60 y 69 puntos), 74 sujetos (51,7\%), estilo de vida bueno (entre 70 y 84 puntos) y 61 sujetos $(42,7 \%)$ estilo de vida excelente (entre 85 y 100 puntos). La confiabilidad total del instrumento evaluada por sexo fue 0,73 , según $\alpha$ de Cronbach. 
Tabla1. Clasificación del cuestionario Fantástico por sexo de la población estudiada, $(n=550)$

\begin{tabular}{lccc}
\hline \multicolumn{1}{c}{ Calificación } & Total & Hombres & Mujeres \\
\hline Malo & $4(0,7)$ & $3(0,7)$ & $1(0,7)$ \\
Regular & $29(5,3)$ & $22(5,4)$ & $7(4,9)$ \\
Bueno & $258(46,9)$ & $184(45,2)$ & $74(51,7)$ \\
Excelente & $259(47,1)$ & $198(48,6)$ & $61(42,7)$ \\
Total & $550(100)$ & $407(100)$ & $143(100)$ \\
\hline
\end{tabular}

Los resultados por media, desviación estándar y $\alpha$ de Cronbach del cuestionario Fantástico-25 ítems- y los agrupados en los 10 dominios: (Familia y amigos, Actividad física, Nutrición, consumo de Tabaco, consumo de Alcohol, Sueño y estrés, Tipo de personalidad, Introspección, Conducción al trabajo, Otras drogas), se muestran en las Tablas 2 y 3.

Tabla 2.Descripción en media, desviación estándar (DE) y ade cronbach de los 25 Ítems del cuestionario Fantástico, $(n=550)$

\begin{tabular}{clrc}
\hline Ref. & \multicolumn{1}{c}{ Pregunta } & $\begin{array}{c}\text { Media } \\
(\mathrm{DE})\end{array}$ & $\begin{array}{c}\alpha \text { de } \\
\text { Cronbach }\end{array}$ \\
\hline 1 & Tengo con quien hablar de las cosas que son importantes para mí. & $1,9(0,2)$ & 0,69 \\
2 & Yo doy y recibido cariño. & $1,9(0,2)$ & 0,69 \\
3 & Yo realizo actividad física (caminar, subir escaleras). & $1,6(0,5)$ & 0,67 \\
4 & Yo hago ejercicio al menos por 20 min, (correr, caminar rápido). & $1,0(0,6)$ & 0,68 \\
5 & Mi alimentación es balanceada. & $1,5(0,5)$ & 0,68 \\
6 & A menudo consumo mucha azúcar, sal, comida chatarra o con grasa. & $1,0(0,4)$ & 0,69 \\
7 & Estoy pasado a mi peso ideal en _ Kg. & $1,6(0,6)$ & 0,69 \\
8 & Yo fumo cigarrillos. & $1,4(0,8)$ & 0,70 \\
9 & Generalmente fumo cigarrillos por día. & $1,8(0,4)$ & 0,68 \\
10 & Mi número promedio de tragos por semana es de & $1,9(0,3)$ & 0,69 \\
11 & Bebo más de cuatro tragos en una misma ocasión. & $1,2(0,5)$ & 0,68 \\
12 & Manejo el auto después de beber alcohol. & $1,8(0,3)$ & 0,69 \\
13 & Duermo bien y me siento descansado. & $1,7(0,5)$ & 0,68 \\
14 & Yo me siento capaz de manejar el estrés o la tensión en mi vida. & $1,8(0,4)$ & 0,68 \\
15 & Yo me relajo y disfruto mi tiempo libre. & $1,8(0,3)$ & 0,69 \\
16 & Parece que ando acelerado. & $1,2(0,5)$ & 0,68 \\
17 & Me siento enojado o agresivo. & $1,6(0,5)$ & 0,67 \\
18 & Yo soy un pensador positivo u optimista. & $1,9(0,2)$ & 0,69 \\
19 & Yo me siento tenso o apretado. & $1,5(0,5)$ & 0,67 \\
20 & Yo me siento deprimido o triste. & $1,7(0,4)$ & 0,68 \\
21 & Uso siempre el cinturón de seguridad. & $1,8(0,4)$ & 0,69 \\
22 & Yo me siento satisfecho con mi trabajo o mis actividades. & $1,9(0,1)$ & 0,69 \\
23 & Uso drogas como marihuana o cocaína. & $1,8(0,1)$ & 0,69 \\
24 & Uso excesivamente medicamentos que me indican o sin receta médica. & $1,8(0,4)$ & 0,69 \\
25 & Bebo café, té o bebidas cola que tienen cafeína. & $1,8(0,3)$ & 0,68 \\
\hline
\end{tabular}


Tabla 3. Descripción en medias y desviación estándar (DE) en los dominios del Cuestionario Fantástico por grupos de edad y sexo

\begin{tabular}{lcccccc}
\hline \multirow{4}{*}{ Dominio } & $\begin{array}{c}\text { Mujeres } \\
\text { Total } \\
(\mathrm{n}=143)\end{array}$ & $\begin{array}{c}\text { Mujeres } \\
18-34 \\
(\mathrm{n}=93)\end{array}$ & $\begin{array}{c}\text { Mujeres } \\
35-64 \\
(\mathrm{n}=50)\end{array}$ & $\begin{array}{c}\text { Hombres } \\
\text { Total } \\
(\mathrm{n}=407)\end{array}$ & $\begin{array}{c}\text { Hombres } \\
18-34 \\
(\mathrm{n}=295)\end{array}$ & $\begin{array}{c}\text { Hombres } \\
35-64 \\
(\mathrm{n}=112)\end{array}$ \\
\cline { 2 - 7 } & $\begin{array}{c}\text { Media } \\
(\mathrm{DE})\end{array}$ & $\begin{array}{c}\text { Media } \\
(\mathrm{DE})\end{array}$ & $\begin{array}{c}\text { Media } \\
(\mathrm{DE})\end{array}$ & $\begin{array}{c}\text { Media } \\
(\mathrm{DE})\end{array}$ & $\begin{array}{c}\text { Media } \\
(\mathrm{DE})\end{array}$ & $\begin{array}{c}\text { Media } \\
(\mathrm{DE})\end{array}$ \\
\hline Familia y amigos & $3,8(0,4)$ & $3,8(0,5)$ & $3,8(0,4)$ & $3,8(0,4)$ & $3,8(0,4)$ & $3,8(0,3)$ \\
Actividad fisica & $2,4(1,2)$ & $2,3(1,2)$ & $2,5(1,2)$ & $2,6(1,0)$ & $2,7(0,9)$ & $2,5(1,1)$ \\
Nutrición & $4,5(1,0)$ & $4,4(1,0)$ & $4,6(1,0)$ & $4,1(1,1)$ & $4,2(1,1)$ & $4,1(1,2)$ \\
Tabaco & $3,5(0,9)$ & $3,5(0,9)$ & $3,5(0,9)$ & $3,2(1,1)$ & $3,1(1,2)$ & $3,5(0,9)$ \\
Alcohol & $5,4(0,8)$ & $5,3(0,8)$ & $5,4(0,6)$ & $4,8(0,9)$ & $4,8(0,9)$ & $4,9(0,9)$ \\
Sueño y estrés & $4,9(1,1)$ & $4,9(1,1)$ & $5,1(1,1)$ & $5,3(0,9)$ & $5,4(0,8)$ & $5,2(1,0)$ \\
Tipo de personalidad & $2,5(1,0)$ & $2,5(0,9)$ & $2,6(1,1)$ & $2,9(0,8)$ & $3,0(0,8)$ & $2,7(1,0)$ \\
Introspección & $4,8(1,1)$ & $4,8(1,0)$ & $4,7(1,2)$ & $5,1(0,9)$ & $5,1(0,9)$ & $5,0(0,9)$ \\
Conducción trabajo & $3,6(0,5)$ & $3,6(0,6)$ & $3,7(0,5)$ & $3,7(0,5)$ & $3,7(0,5)$ & $3,8(0,4)$ \\
Otras drogas & $5,5(0,7)$ & $5,5(0,7)$ & $5,4(0,6)$ & $5,6(0,6)$ & $5,6(0,6)$ & $5,5(0,7)$ \\
Total & $82,7(8,9)$ & $82,1(8,9)$ & $83,6(8,9)$ & $83,5(8,6)$ & $83,6(8,5)$ & $83,1(8,6)$ \\
\hline
\end{tabular}

La consistencia interna por dominio del instrumento Fantástico por grupos de edad y sexo, se presentan en la Tabla 4. Todos los dominios del cuestionario contribuyen a la estabilidad y confiabilidad del instrumento, $\alpha$ de Cronbach $>0,50$.

Por último, en las Tablas 5 y 6 , se muestra la correlación interescalar de los dominios del cuestionario FANTÁSTICO estimada a través del coeficiente de correlación de Pearson (r). En mujeres y hombres, se encontraron correlaciones consideradas como buenas y aceptables, en las diferentes categorías del cuestionario Fantástico (total vs. dominios), $\mathrm{p}<0,01$.

Tabla 4. Coeficientes de confiabilidad $\alpha$ de Cronbach en los dominios del Cuestionario Fantástico por grupos de edad y sexo

\begin{tabular}{ccccccccccc}
\hline Características & $\mathrm{F}$ & $\mathrm{A}$ & $\mathrm{N}$ & $\mathrm{T}$ & $\mathrm{A}$ & $\mathrm{S}$ & $\mathrm{T}$ & $\mathrm{I}$ & $\mathrm{C}$ & $\mathrm{O}$ \\
\hline Total $(\mathrm{n}=550)$ & 0,54 & 0,52 & 0,52 & 0,53 & 0,53 & 0,51 & 0,51 & 0,51 & 0,54 & 0,54 \\
Grupos de edad & & & & & & & & & & \\
$\quad 18-34(\mathrm{n}=388)$ & 0,51 & 0,50 & 0,54 & 0,52 & 0,50 & 0,50 & 0,50 & 0,54 & 0,53 & 0,57 \\
$35-64(\mathrm{n}=162)$ & 0,51 & 0,50 & 0,54 & 0,53 & 0,55 & 0,50 & 0,49 & 0,54 & 0,53 & 0,56 \\
Sexo & & & & & & & & & & \\
$\quad$ Mujeres $(\mathrm{n}=143)$ & 0,54 & 0,51 & 0,53 & 0,54 & 0,55 & 0,50 & 0,51 & 0,50 & 0,55 & 0,54 \\
$\quad$ Hombres $(\mathrm{n}=407)$ & 0,55 & 0,53 & 0,51 & 0,53 & 0,53 & 0,52 & 0,52 & 0,51 & 0,54 & 0,54 \\
\hline
\end{tabular}


Tabla 5. Correlación Interescalar de los dominios del Cuestionario Fantástico en mujeres y grupos de edad, $(n=143)$

\begin{tabular}{|c|c|c|c|c|c|c|c|c|c|c|c|}
\hline \multicolumn{12}{|c|}{ Mujeres Total } \\
\hline Dominio & $\mathrm{F}$ & A & $\mathrm{N}$ & $T$ & A & $S$ & $T$ & I & C & 0 & Total \\
\hline $\mathrm{F}$ & 1 & & & & & & & & & & \\
\hline A & 0,12 & 1 & & & & & & & & & \\
\hline $\mathrm{N}$ & 0,06 & 0,27 & 1 & & & & & & & & \\
\hline $\mathrm{T}$ & 0,08 & 0,05 & 0,01 & 1 & & & & & & & \\
\hline A & 0,04 & 0,05 & $-0,03$ & 0,06 & 1 & & & & & & \\
\hline $\mathrm{S}$ & $0,22^{*}$ & $0,37^{*}$ & $0,20^{*}$ & 0,03 & $-0,02$ & 1 & & & & & \\
\hline T & $0,26^{*}$ & 0,15 & 0,09 & 0,02 & 0,03 & $0,26^{*}$ & 1 & & & & \\
\hline I & $0,20^{*}$ & $0,30^{*}$ & $0,15^{*}$ & 0,02 & 0,09 & $0,45^{*}$ & $0,53^{*}$ & 1 & & & \\
\hline C & 0,04 & $-0,01$ & $-0,00$ & 0,02 & 0,00 & 0,08 & 0,12 & $0,18^{*}$ & 1 & & \\
\hline $\mathrm{O}$ & $0,27^{*}$ & 0,08 & $0,15^{\star}$ & 0,07 & 0,07 & $0,17^{*}$ & $0,20^{*}$ & $0,25^{*}$ & 0,03 & 1 & \\
\hline Total & $0,40^{*}$ & 0,59 & $0,44^{*}$ & $0,29^{*}$ & $0,24^{*}$ & 0,65 & $0,58^{*}$ & $0,71^{*}$ & $0,23^{*}$ & 0,43 & 1 \\
\hline \multicolumn{12}{|c|}{ Mujeres $18-34$ años } \\
\hline $\mathrm{F}$ & 1 & & & & & & & & & & \\
\hline A & 0,06 & 1 & & & & & & & & & \\
\hline $\mathrm{N}$ & 0,07 & $0,29^{*}$ & 1 & & & & & & & & \\
\hline T & $0,13^{*}$ & 0,09 & 0,10 & 1 & & & & & & & \\
\hline A & 0,01 & 0,03 & $-0,18^{*}$ & 0,09 & 1 & & & & & & \\
\hline S & $0,17^{*}$ & 0,40 & 0,11 & $0,14^{*}$ & 0,02 & 1 & & & & & \\
\hline $\mathrm{T}$ & $0,12^{*}$ & $0,27^{*}$ & 0,11 & $0,14^{*}$ & 0,06 & $0,21^{*}$ & 1 & & & & \\
\hline I & $0,17^{\star}$ & $0,33^{*}$ & 0,10 & 0,07 & $0,19^{*}$ & $0,40^{*}$ & $0,59^{*}$ & 1 & & & \\
\hline C & $-0,08$ & 0,01 & 0,00 & 0,05 & 0,00 & 0,06 & 0,07 & $0,14^{*}$ & 1 & & \\
\hline $\mathrm{O}$ & $0,22^{*}$ & 0,08 & $0,13^{*}$ & $0,12^{*}$ & 0,08 & $0,17^{*}$ & $0,18^{*}$ & $0,22^{*}$ & 0,06 & 1 & \\
\hline Total & $0,32^{*}$ & $0,63^{*}$ & $0,40^{*}$ & $0,40^{*}$ & $0,26^{*}$ & $0,62^{*}$ & $0,60^{*}$ & $0,71^{*}$ & $0,22^{*}$ & $0,43^{*}$ & 1 \\
\hline \multicolumn{12}{|c|}{ Mujeres $35-64$ años } \\
\hline $\mathrm{F}$ & 1 & & & & & & & & & & \\
\hline A & $0,24^{*}$ & 1 & & & & & & & & & \\
\hline $\mathrm{N}$ & 0,06 & $0,22^{*}$ & 1 & & & & & & & & \\
\hline $\mathrm{T}$ & $-0,01$ & $-0,02$ & $-0,15^{*}$ & 1 & & & & & & & \\
\hline A & 0,11 & 0,12 & 0,28 & 0,00 & 1 & & & & & & \\
\hline $\mathrm{S}$ & $0,33^{*}$ & $0,32^{*}$ & $0,33^{*}$ & $-0,14^{*}$ & $-0,15^{*}$ & 1 & & & & & \\
\hline$T$ & $0,51^{*}$ & $-0,00$ & 0,06 & $-0,13^{*}$ & $-0,02$ & $0,33^{*}$ & 1 & & & & \\
\hline I & $0,26^{*}$ & 0,25 & $0,23^{*}$ & $-0,06$ & $-0,11$ & $0,54^{*}$ & $0,45^{*}$ & 1 & & & \\
\hline $\mathrm{C}$ & $0,32^{*}$ & $-0,08$ & $-0,06$ & $-0,03$ & $-0,01$ & 0,10 & $0,19^{*}$ & $0,25^{*}$ & 1 & & \\
\hline $\mathrm{O}$ & $0,39^{*}$ & 0,10 & $0,20^{*}$ & $-0,02$ & 0,06 & $0,19^{*}$ & $0,22^{*}$ & $0,32^{*}$ & $-0,13^{*}$ & 1 & \\
\hline Total & $0,59^{*}$ & $0,52^{*}$ & $0,49^{*}$ & $0,07^{*}$ & 0,19 & 0,68 & $0,57^{*}$ & $0,79^{*}$ & $0,25^{*}$ & 0,46 & 1 \\
\hline
\end{tabular}

Correlación bilateral (r pearson), ${ }^{*} p<0,01$ 
Tabla 6. Correlación Interescalar de los dominios del Cuestionario Fantástico en hombres y grupos de edad, $(n=407)$

\begin{tabular}{|c|c|c|c|c|c|c|c|c|c|c|c|}
\hline \multicolumn{12}{|c|}{ Hombres Total } \\
\hline Dominio & $\mathrm{F}$ & A & $\mathrm{N}$ & $T$ & A & $\mathrm{S}$ & $T$ & I & C & $\mathrm{O}$ & Total \\
\hline $\mathrm{F}$ & 1 & & & & & & & & & & \\
\hline A & 0,09 & 1 & & & & & & & & & \\
\hline $\mathrm{N}$ & 0,05 & 0,25 & 1 & & & & & & & & \\
\hline $\mathrm{T}$ & 0,07 & 0,07 & 0,12 & 1 & & & & & & & \\
\hline A & 0,08 & 0,05 & 0,13 & 0,15 & 1 & & & & & & \\
\hline S & $0,27^{*}$ & 0,07 & 0,17 & $-0,00$ & $0,18^{*}$ & 1 & & & & & \\
\hline $\mathrm{T}$ & 0,14 & 0,13 & 0,16 & 0,04 & $0,21^{*}$ & $0,37^{*}$ & 1 & & & & \\
\hline I & $0,21^{*}$ & 0,07 & 0,18 & $-0,00$ & $0,25^{*}$ & $0,45^{*}$ & $0,49^{*}$ & 1 & & & \\
\hline C & 0,11 & 0,01 & 0,05 & 0,17 & 0,12 & $0,21^{*}$ & 0,11 & $0,16^{*}$ & 1 & & \\
\hline $\mathrm{O}$ & 0,10 & 0,01 & 0,15 & 0,10 & 0,09 & $0,26^{*}$ & $0,21^{*}$ & $0,19^{*}$ & 0,13 & 1 & \\
\hline Total & $0,35^{*}$ & $0,42^{*}$ & $0,54^{*}$ & $0,40^{*}$ & $0,48^{*}$ & $0,59^{*}$ & $0,59^{*}$ & $0,61^{*}$ & $0,65^{*}$ & $0,41^{*}$ & 1 \\
\hline \multicolumn{12}{|c|}{ Hombres $18-34$ años } \\
\hline $\mathrm{F}$ & 1 & & & & & & & & & & \\
\hline A & 0,12 & 1 & & & & & & & & & \\
\hline $\mathrm{N}$ & 0,06 & 0,20 & 1 & & & & & & & & \\
\hline $\mathrm{T}$ & 0,07 & 0,05 & 0,10 & 1 & & & & & & & \\
\hline A & 0,09 & 0,05 & 0,13 & 0,17 & 1 & & & & & & \\
\hline S & $0,25^{*}$ & 0,05 & $0,16^{*}$ & $-0,00$ & $0,22^{*}$ & 1 & & & & & \\
\hline $\mathrm{T}$ & 0,11 & 0,15 & 0,17 & 0,11 & $0,31^{*}$ & $0,34^{*}$ & 1 & & & & \\
\hline I & $0,22^{*}$ & 0,10 & $0,18^{*}$ & 0,02 & $0,25^{*}$ & $0,43^{*}$ & $0,47^{*}$ & 1 & & & \\
\hline C & 0,06 & 0,02 & 0,06 & 0,15 & 0,15 & $0,21^{*}$ & 0,14 & 0,15 & 1 & & \\
\hline $\mathrm{O}$ & 0,16 & 0,09 & $0,21^{*}$ & 0,12 & 0,15 & $0,26^{*}$ & $0,18^{*}$ & $0,20^{*}$ & $0,13^{*}$ & 1 & \\
\hline Total & $0,35^{*}$ & $0,41^{*}$ & $0,52^{*}$ & $0,43^{*}$ & $0,52^{*}$ & $0,56^{*}$ & $0,61^{*}$ & $0,61^{*}$ & $0,36^{*}$ & $0,46^{*}$ & 1 \\
\hline \multicolumn{12}{|c|}{ Hombres $35-64$ años } \\
\hline $\mathrm{F}$ & 1 & & & & & & & & & & \\
\hline A & 0,01 & 1 & & & & & & & & & \\
\hline $\mathrm{N}$ & 0,06 & $0,36^{*}$ & 1 & & & & & & & & \\
\hline $\mathrm{T}$ & 0,02 & $0,17^{*}$ & $0,19^{*}$ & 1 & & & & & & & \\
\hline A & 0,04 & 0,05 & $0,15^{*}$ & 0,09 & 1 & & & & & & \\
\hline S & $0,38^{*}$ & 0,11 & $0,21^{*}$ & 0,05 & 0,08 & 1 & & & & & \\
\hline $\mathrm{T}$ & $0,26^{*}$ & 0,08 & $0,16^{*}$ & $-0,03^{*}$ & 0,02 & $0,41^{*}$ & 1 & & & & \\
\hline I & $0,22^{*}$ & $-0,01$ & $0,17^{*}$ & $-0,07$ & $0,23^{*}$ & $0,47^{*}$ & $0,54^{*}$ & 1 & & & \\
\hline C & $0,30^{*}$ & 0,02 & 0,02 & $0,24^{*}$ & 0,04 & $0,22^{*}$ & 0,07 & $0,23^{*}$ & 1 & & \\
\hline $\mathrm{O}$ & $-0,04$ & $-0,15^{*}$ & 0,01 & $0,13^{*}$ & $-0,01$ & $0,24^{*}$ & $0,25^{*}$ & 0,16 & 0,16 & 1 & \\
\hline Total & $0,35^{*}$ & $0,44^{*}$ & $0,59^{*}$ & $0,37^{*}$ & $0,38^{*}$ & $0,65^{*}$ & $0,59^{*}$ & $0,60^{*}$ & $0,35^{*}$ & $0,31^{*}$ & 1 \\
\hline
\end{tabular}

Correlación bilateral (r pearson), ${ }^{* *} p<0,01$

\section{DISCUSIÓN}

La medición del constructo "estilo de vida" es una tarea difícil debido a las múltiples dimensiones que lo componen y debido a las dificultades que implica su medición directa de manera objetiva. El cuestionario Fantástico, fue incorporado en Canadá, al Plan de Evaluación de la Actividad Física, Fitness y Estilo de Vida en 1996, con el objetivo de caracterizar y medir el estilo de vida (5). Varios estudios han concluido que este cuestionario tiene validez del constructo, validez de contenido, coherencia y buen nivel para determinar el estilo de vida de personas saludables y sujetos con enfermedades crónicas (8-13). 
La consistencia interna de los ítems y de los dominios medida por el alfa de Cronbach fue mayor a0,50,pero menor que la obtenida por Rodríguez-Moctezuma et al (8) y Rodríguez et al (9) en diabéticos tipo 2 y en población saludable $(\alpha=\approx 0,80)$ y $(\alpha=\approx 0,70)$, respectivamente. De acuerdo con Hill y Hill (19), "un $\alpha$ de Cronbach entre 0,7 y 0,8 se puede considerar aceptable, y la validez interna de un instrumento diseñado para medir una variable latente que sólo puede ser estimado y no medido". Este postulado aplica a instrumentos y cuestionarios que recogen un conjunto de datos empíricos de una muestra de personas. De esta manera los coeficientes de fiabilidad, como en la mayoría de las estadísticas descriptivas varían de una muestra a otra y tienen que ser consideradas como estimaciones. Otros autores afirman que un $\alpha$ de Cronbach superior a 0,5 , en estudios de validación de cuestionarios puede ser considerado como aceptable $(16,19)$.

Todos los ítems del cuestionario Fantástico han presentado aceptable y buena consistencia interna por el $\alpha$ de Cronbach, superior a 0,67 ítemtotal, total general $>0,51$,por grupos de edad $>0,49$, y por sexo $>0,50$. El análisis de las preguntas, agrupadas en los dominios, permite observar que todos ellos están contribuyendo a la estabilidad del instrumento. El a de Cronbach entre los ítems fue $>0,67$,(Tabla 2).

Cuando se consideran las cuatro categorías de clasificación (malo, regular, bueno y excelente), se encontró que 4 sujetos $(0,7 \%)$ obtuvieron una calificación de malo (inferior a 60 puntos), 29 sujetos (5,3\%), regular (entre 60 y 69 puntos), 258 sujetos (47\%) bueno (entre 70 y 84 puntos) y 259 sujetos ( $47 \%$ ) excelente (entre 85 y 100 puntos). En este grupo, la confiabilidad del instrumento evaluada por el $\alpha$ de Cronbach fue 0,73. Al comparar por sexo esta clasificación se observa una tendencia similar a la reportada en población general.

Los resultados de este estudio sugieren que el instrumento posee una buena capacidad de calificación. Si hay cambios en las variables que determinan el estilo de vida variable latente, la gente estará debidamente reclasificada. Esto es especialmente importante para los programas de intervención, cuyo propósito es promover el estilo de vida como una forma de mejorar la salud y la calidad de vida. Cambios positivos en la clasificación según el cuestionario Fantástico indicará que el programa de intervención está alcanzando los resultados esperados. 
Es importante recordar que la aplicación del cuestionario Fantástico de manera auto-diligenciada será válida solo para adultos aparentes saludables, siendo ésta la principal limitación del estudio. Sin embargo, el Plan Canadiense de Evaluación de la Actividad Física, Fitness y estilo de vida, ha sugerido el uso del cuestionario a partir de los 15 años. El impacto de este estudio es la validación de un instrumento destinado a evaluar los principales elementos que caracterizan el estilo de vida adecuado para la salud (20). En conclusión la versión Colombiana del cuestionario Fantástico reúne algunos de los criterios de consistencia interna y validez de constructo. Se propone como una herramienta fácil y sencilla para evaluar el estilo de vida de los adultos

Conflicto de intereses: Ninguno

\section{REFERENCIAS}

1. World Health Organization (WHO). Health promotion glossary. Geneva:WHO papers; 1998.

2. Blair SN, Horton E, Leon AS, Lee I-MIN, Drinkwater BL, Dishman RK, et al. Physical activity, nutrition, and chronic disease. Med Sci Sports Exerc. 1996;28:335-49.

3. USDHHS - U.S. Department of Health and Human Services. Physical Activity and Health: a Report of the Surgeon General. Atlanta, GA: U.S. Department of Health and Human Services, Center for Disease Control and Prevention, National Center for Chronic Disease Prevention and Health Promotion; 1996.

4. U.S. Department of Health and Human Services. Health-related quality of life and activity limitation-eight states. MMWR. 1998;47:135-40.

5. CSEF-Canadian Society for Exercise Physiology. The Canadian Physical Activity, Fitness and Lifestyle Appraisal: CSEP's guide to health active living. 2nd ed. Ottawa: CSEF; 2003.

5. Reaven GM. Banting lecture 1988. Role of insulin resistance in human disease. Diabetes. 1988;37:1595-607.

7. López-Carmona JM, Rodríguez-Moctezuma JR, Munguia Miranda C, HernándzezSantiago JL, Casas de La Torre E. Validez y fiabilidad del instrumento FANTASTIC para medir el estilo de vida de pacientes mexicanos con hipertensión arterial. Aten Primaria. 2000;26:542-9.

8. Rodriguez-Moctezuma R, López-Carmona JM, Munguia Miranda C, Hernández-Santiago JL, Bermúdez-Martínez M. Validez y consistencia del instrumento "FANTASTIC" para medir estilo de vida en diabéticos. Rev Med IMSS. 2003;41:211-20.

9. Rodríguez CR, Reis RS, Petroski EL. Brazilian Version of a Lifestyle Questionnaire: Translation and Validation for Young Adults. Arq Bras Cardiol. 2008;91:92-8.

10. Wilson DM, Ciliska D. Life-style assessment: Development and use of the FANTASTIC checklist. Can Fam Physician. 1984;30:1527-32.

11. Wilson DM, Ciliska D. Life-style assessment: Helping patients change health behaviors. Can Fam Physician. 1984;30:1665-70.

12. Wilson DM, Ciliska D. Lifestyle assessment: Testing the FANTASTIC instrument. Can Fam Physician. 1984;30:1863-6.

13. Kamien M, Power R. Lifestyle and health habits of fourth year medical students a University of Western Australia. Aus Fam Physician. 1996;Supl I:26-29.

14. Sharratt JK, Sharratt MT, Smith DM, Howell MJ, Davenport L. FANTASTIC lifestyle survey 
of University of Waterloo Employes. Can Fam Physician. 1984;30:1869-72.

15. Kason Y, Ylanko V. FANTASTIC life-style assessment: Part 5. Measuring lifestyle in family practice. Can Fam Physician. 1984;30:2379-83.

16. Hulme PA, Walker SN, Effle KJ, Jorgensen L, McGowan MG, Nelson JD, Pratt EN. Healthpromoting lifestyle behaviors of Spanish-speaking Hispanic adults. J Transcult Nurs. 2003;14:244-54.

17. López-Carmona JM, Ariza-Andraca CR, Rodríguez-Moctezuma JR, Monguíra-Miranda C. Construcción y validación inicial de un instrumento para medir el estilo de vida en pacientes con diabetes mellitus tipo 2. Salud Pública Mex. 2003;45:259-68.

18. Hair JF, Anderson RE, Tatham RL, Black WC. Análisis multivariante. $5^{a}$ ed. Madrid: Prentice Hall; 1999.

19. Hill MM, Hill A. Investigação por questionário. Lisboa: Silabo; 2000.

20. Triviño-Quintero LP, Dosman-González V, Uribe-Vélez YL, Agredo-Zuñiga RA, JerezValderrama AM, Ramírez-Vélez R. Estudio del estilo de vida y su relación con factores de riesgo cardiovascular en adultos de mediana edad. Acta Med Colomb. 2009;34:158-63. 